\title{
THE HARNACK INEQUALITY FOR SECOND-ORDER ELLIPTIC EQUATIONS WITH DIVERGENCE-FREE DRIFTS*
}

\author{
MIHAELA IGNATOVA ${ }^{\dagger}$, IGOR KUKAVICA ${ }^{\ddagger}$, AND LENYA RYZHIK ${ }^{\S}$
}

\begin{abstract}
We consider an elliptic equation with a divergence-free drift $b$. We prove that an inequality of Harnack type holds under the assumption $b \in L^{n / 2+\delta}$ where $\delta>0$. As an application we provide a one-sided Liouville's theorem provided that $b \in L^{n / 2+\delta}\left(\mathbb{R}^{n}\right)$.
\end{abstract}

Key words. Harnack inequality, Liouville theorem, regularity, drift-diffusion equations.

AMS subject classifications. 35B53, 35B65, 35J15, 35Q35.

\section{Introduction}

In this paper, we consider elliptic equations of the form

$$
-\Delta u+b \cdot \nabla u+a u=0
$$

in a domain $\Omega \subset \mathbb{R}^{n}$. Here $a(x)$ is a given function and $b(x)$ is a prescribed divergence free vector field, i.e., $\operatorname{div} b=0$. The qualitative properties of solutions to elliptic and parabolic equations in divergence form with low regularity of the coefficients have been studied extensively, starting with the classical papers of De Giorgi [5], Nash [12], and Moser [11]. We are mostly interested in the improved regularity for divergence free drifts $b$, which arise in fluid dynamics models (cf. $[1,2,6,9,15,10,16]$ ).

As can be easily seen from a simple scaling argument, the natural Lebesgue spaces for the coefficients in the equation for the local regularity theory to hold are $a \in L^{n / 2}$ and $b \in L^{n}$, and, indeed, regularity properties of solutions for the case $a \in L^{n / 2+\delta}$, where $\delta>0$, and $b \in L^{n}$ have been known since the work of Stampacchia [14]. It is well known that a strong divergence free flow may induce better regularity and decay of solutions of elliptic and parabolic problems by means of improved mixing; see, for instance, [4] and references therein. It is also known that a divergence free-drift of critical regularity can still lead to regular solutions [2,3]. The question we study in this paper is whether the divergence free condition on $b$ allows one to relax the regularity assumptions on $b$ given by Stampacchia.

Let us recall some recent results in this direction. In a recent paper [13], Nazarov and Ural'tseva significantly relaxed the classical regularity assumptions for divergencefree $b$ by establishing the Harnack inequality and the Liouville theorem for weak solutions to (1.1) if $b$ belongs to a Morrey space $M_{q}^{n / q-1}$ with $n / 2<q \leq n$, which lies between $L^{n}$ and $B M O^{-1}$. In [6], Friedlander and Vicol proved the Hölder continuity of weak solutions to drift-diffusion equations with a drift in $B M O^{-1}$. In [15], Seregin et al. established the Liouville theorem and the Harnack inequality for elliptic and parabolic equations with divergence free drifts $b$ lying in the scale invariant space

*Received: July 9, 2012; accepted (in revised form): May 20, 2013. Communicated by Alexander Kiselev.

${ }^{\dagger}$ Department of Mathematics, Stanford University, Stanford, CA 94305, USA (mihaelai@stanford. edu).

${ }^{\ddagger}$ Department of Mathematics, University of Southern California, Los Angeles, CA 90089, USA (kukavica@usc.edu).

$\S$ Department of Mathematics, Stanford University, Stanford, CA 94305, USA (ryzhik@stanford. edu). 
$B M O^{-1}$. All these spaces share the same scaling properties as $L^{n}$ and are thus the natural candidates for good regularity theory.

In the present paper, we establish the Harnack inequality and the one-sided Liouville theorem for Lipschitz generalized solutions to (1.1) when $a(x)$ and $b(x)$ lie in the space $L^{q}(\Omega)$ with $n / 2<q \leq n$, and $b$ is divergence free. Our results also hold for weak solutions provided that the drift $b$ satisfies certain additional assumptions (cf. equation (27) in [13]). More precisely, we establish a Harnack-type inequality

$$
\sup _{y \in B_{R}(x)} u(y) \leq C \inf _{y \in B_{R}(x)} u(y),
$$

for all $R>0$ (see Theorem 2.1), and use this estimate to establish the one-sided Liouville theorem when $a=0$ in Theorem 2.3. The constant $C$ in (1.2) depends on the $L^{q}$-norms of $a$ and $b$, where $q>n / 2$, but not on the solution $u$. Note that the $L^{n / 2}$-norm is not scale invariant: If we set $b_{l}(x)=(1 / l) b(x / l)$, then $\left\|b_{l}\right\|_{L^{n / 2}}=l\|b\|_{L^{n / 2}}$. Because of this, one can not expect the constant $C$ to be independent of $R$, and, indeed, the constant given explicitly in Remark 2.2 blows up as $R \rightarrow 0$.

The paper is organized as follows. In Section 2, we state our main results, Theorems 2.1 and 2.3. The proof is based on two auxiliary results, Lemmas 2.4 and 2.5. We first show (see Lemma 2.4) that weak solutions of (1.1) are locally bounded by employing the classical Moser iteration technique. Then, in Lemma 2.5, we derive a weak Harnack inequality, the proof of which is inspired by the proof of Han and Lin [8, Theorem 4.15] for elliptic equations without lower-order coefficients. Our main results, Theorems 2.1 and 2.3, are direct consequences of Lemmas 2.4 and 2.5.

\section{The main results}

Our first result is the Harnack inequality.

THEOREM 2.1 (Harnack inequality). Let $u$ be a nonnegative Lipschitz solution to the elliptic equation (1.1) in $\Omega$, that is,

$$
\int_{\Omega}\left(\partial_{j} u\right)\left(\partial_{j} \varphi\right)+\int_{\Omega} b_{j}\left(\partial_{j} u\right) \varphi+\int_{\Omega} a u \varphi=0
$$

for any Lipschitz function $\varphi \geq 0$ in $\Omega$ such that $\varphi=0$ in $\Omega^{c}$. Assume that $a \in L^{q}(\Omega)$, $b \in L^{\bar{q}}(\Omega)$ for $n / 2<q, \bar{q} \leq n$, and that $\operatorname{div} b=0$ in the sense of distributions. Then for any $B_{R} \subset \Omega$ we have

$$
\sup _{B_{R}} u \leq C \inf _{B_{R}} u .
$$

Here $C$ is a constant depending on $n, q, \bar{q}, R,\|a\|_{L^{q}\left(B_{R}\right)}$, and $\|b\|_{L^{\bar{q}}\left(B_{R}\right)}$.

REMARK 2.2. From the proof we can deduce that

$$
\begin{aligned}
C=C(n, q, \bar{q})\left(1+\left(R^{2-n / q}\|a\|_{L^{q}\left(B_{R}\right)}\right)^{1 /(2-n / q)}\right. & \\
& \left.+\left(R^{1-n / \bar{q}}\|b\|_{L^{\bar{q}}\left(B_{R}\right)}\right)^{1 /(2-n / \bar{q})}\right)^{C(n) M_{R}},
\end{aligned}
$$

where $M_{R}=1+R^{2-n / q}\|a\|_{L^{q}\left(B_{R}\right)}+R^{1-n / \bar{q}}\|b\|_{L^{\bar{q}}\left(B_{R}\right)}$.

Theorem 2.1 has the following consequence when $\Omega=\mathbb{R}^{n}$. 
Theorem 2.3 (One-sided Liouville's theorem). Let $a(x) \equiv 0$ and $b(x)$ be as in Theorem 2.1. Then any nonnegative Lipschitz solution $u$ to the elliptic equation (1.1) in $\mathbb{R}^{n}$ is equal to a constant.

We note that [13] provides a two-sided Liouville's theorem under the same assumptions, that is, the only solutions of (1.1) that are bounded both from above and from below are constants. However, the one-sided Liouville's theorem in [13] requires $b$ to belong to a Morrey space which is in the same scaling class as $L^{n}$.

Proof. Without loss of generality, we may assume that $u$ is a nonnegative Lipschitz solution to (1.1) with $\inf _{\mathbb{R}^{n}} u=0$. Then for every $\epsilon>0$, we have $\inf _{B_{R}} u \leq \epsilon$ for any sufficiently large ball $B_{R}$. By Theorem 2.1, $\sup _{B_{R}} u \leq C \inf _{B_{R}} u \leq C \epsilon$ for all sufficiently large $R>0$. Observe that the constant $C$ given explicitly by (2.2) depends on $R$ but remains bounded as $R \rightarrow \infty$. Therefore, the assertion is established.

Theorem 2.1 is an immediate consequence of the following two lemmas that compare $\sup _{B_{\theta R}} u$ and $\inf _{B_{\theta R}} u$ to $\|u\|_{L^{p}\left(B_{\tau R}\right)}$ with some small $p>0$ and $0<\theta<\tau<1$. Here, for $0<p<1$ we still use the notation

$$
\|u\|_{L^{p}(B)}=\left(\int_{B} u^{p}\right)^{1 / p}
$$

though it is not a norm.

Lemma 2.4. Assume that $u$ is a nonnegative Lipschitz subsolution to the equation

$$
-\Delta u+b \cdot \nabla u+a u=0
$$

with $a \in L^{q}(\Omega), b \in L^{\bar{q}}(\Omega)$ for $n / 2<q, \bar{q} \leq n$, and $\operatorname{div} b \leq 0$ in the sense of distributions. Then for any $B_{R} \subset \Omega, p>0$, and $0<\theta<\tau<1$,

$$
\begin{aligned}
\sup _{B_{\theta R}} u \leq C(1+ & \left.\left(R^{2-n / q}\|a\|_{L^{q}\left(B_{R}\right)}\right)^{1 /\left(2-\gamma_{0}\right)}+\left(R^{1-n / \bar{q}}\|b\|_{L^{\bar{q}}\left(B_{R}\right)}\right)^{1 /\left(2-\gamma_{0}\right)}\right)^{n / p} \\
& \times R^{-n / p}\|u\|_{L^{p}\left(B_{\tau R}\right)}
\end{aligned}
$$

where $C=C(n, p, \bar{q}, \theta, \tau)$ is a positive constant and $\gamma_{0} \in(0,2)$ is such that $\gamma_{0}=n / \bar{q}$ for $n \geq 3$ and $\gamma_{0}=\left(\bar{q}\left(1 / 2-1 / 2^{*}\right)\right)^{-1}$ for $n=2$ with $2^{*}>2 \bar{q} /(\bar{q}-1)$.

Lemma 2.5. Assume that $u$ is a nonnegative Lipschitz supersolution to (1.1) satisfying the assumptions of Theorem 2.1. Then for any $B_{R} \subset \Omega$ and $0<\theta<\tau<1$ there exists a small positive number $p_{0}=p_{0}\left(n, q, \bar{q}, \theta, \tau, R, M_{R}\right)$ such that

$$
\left(\int_{B_{\tau R}} u^{p_{0}}\right)^{1 / p_{0}} \leq C \inf _{B_{\theta R}} u,
$$

where $C=C\left(n, q, \bar{q}, \theta, \tau, R, M_{R}\right)$ is a positive constant and

$$
M_{R}=1+R^{2-n / q}\|a\|_{L^{q}\left(B_{R}\right)}+R^{1-n / \bar{q}}\|b\|_{L^{\bar{q}}\left(B_{R}\right)} .
$$

Proof. [Proof of Theorem 2.1.] We apply Lemmas 2.4 and 2.5, which are valid for any Lipschitz solution $u \geq 0$ to (1.1) and a small number $p=p_{0}$ as in Lemma 2.5. 
The rest of the paper contains the proofs of Lemma 2.4 and Lemma 2.5. Both lemmas are proved using the Moser iteration, with the general strategy based on the proof of the Harnack inequality in [8].

\section{The proof of Lemma 2.4}

Let $u$ be a nonnegative Lipschitz subsolution of (2.3) in $\Omega$, i.e.,

$$
\int_{\Omega}\left(\partial_{j} u\right)\left(\partial_{j} \varphi\right)+\int_{\Omega} b_{j}\left(\partial_{j} u\right) \varphi+\int_{\Omega} a u \varphi \leq 0
$$

for any Lipschitz function $\varphi \geq 0$ in $\Omega$ such that $\varphi=0$ in $\Omega^{c}$.

For simplicity of presentation, we assume $a=0$. Also, we assume without loss of generality that $R=1$. The proof consists of a priori estimates which can be made rigorous as in [7, 8]. First, we obtain an a priori bound on the $L^{p_{1}}$-norm of $u$ on a smaller ball $B_{r_{1}}$, in terms of an $L^{p_{2}}$-norm of $u$ on a larger ball $B_{r_{2}}$ with $r_{1}<r_{2}$ but $p_{1}>p_{2}$. Then an iterative procedure is used to bring the gap between $r_{1}$ and $r_{2}$ to zero and simultaneously send $p_{1}$ to infinity.

Let $\beta \geq 0$ and $\eta(x)$ be a Lipschitz cut-off in the ball $B_{\tau}$ such that $0 \leq \eta(x) \leq 1$. We use $(\beta / 2+1) u^{\beta+1} \eta^{2 \gamma}$ as a test function in (3.1) to obtain

$$
\begin{aligned}
\left(\frac{\beta}{2}+1\right) \int\left(\partial_{j} u\right) \partial_{j}\left(u^{\beta+1}\right) \eta^{2 \gamma}+\left(\frac{\beta}{2}+1\right) \int u^{\beta+1}\left(\partial_{j} u\right) \partial_{j}\left(\eta^{2 \gamma}\right) \\
+\left(\frac{\beta}{2}+1\right) \int b_{j} u^{\beta+1}\left(\partial_{j} u\right) \eta^{2 \gamma} \leq 0 .
\end{aligned}
$$

Let $w=u^{\beta / 2+1}$ so that $\partial_{j} w=(\beta / 2+1) u^{\beta / 2} \partial_{j} u$. By (3.2), we get

$$
\frac{\beta+1}{\beta / 2+1} \int|\nabla w|^{2} \eta^{2 \gamma} \leq-2 \gamma \int w\left(\partial_{j} w\right) \eta^{2 \gamma-1}\left(\partial_{j} \eta\right)-\int b_{j} w\left(\partial_{j} w\right) \eta^{2 \gamma} .
$$

For the first term in the right side we have

$$
-2 \gamma \int w\left(\partial_{j} w\right) \eta^{2 \gamma-1} \partial_{j} \eta=\gamma \int w^{2}\left(\eta^{2 \gamma-1} \Delta \eta+(2 \gamma-1) \eta^{2 \gamma-2}|\nabla \eta|^{2}\right),
$$

while for the second

$$
-\int b_{j} w\left(\partial_{j} w\right) \eta^{2 \gamma}=\frac{1}{2} \int\left(\partial_{j} b_{j}\right) w^{2} \eta^{2 \gamma}+\gamma \int b_{j} w^{2} \eta^{2 \gamma-1} \partial_{j} \eta \leq \gamma \int b_{j} w^{2} \eta^{2 \gamma-1} \partial_{j} \eta,
$$

as $\operatorname{div} b \leq 0$. Therefore, we get

$$
\begin{gathered}
\int|\nabla w|^{2} \eta^{2 \gamma} \leq \frac{\beta / 2+1}{\beta+1} \gamma \int w^{2}\left(\eta^{2 \gamma-1} \Delta \eta+(2 \gamma-1) \eta^{2 \gamma-2}|\nabla \eta|^{2}\right) \\
+\frac{\beta / 2+1}{\beta+1} \gamma \int b_{j} w^{2} \eta^{2 \gamma-1} \partial_{j} \eta .
\end{gathered}
$$

Next, set $\gamma_{0}=n / \bar{q}$. Then, as $\bar{q}>n / 2$, we have $\gamma_{0} \in(0,2)$ and, in addition

$$
\frac{1}{\bar{q}}+\frac{\gamma_{0}}{2^{*}}+\frac{2-\gamma_{0}}{2}=1
$$


for $n \geq 3$ where $2^{*}=2 n /(n-2)$. If $n=2$ then we may choose $2^{*}>2 \bar{q} /(\bar{q}-1)$ arbitrarily and $\gamma_{0}=\left(\bar{q}\left(1 / 2-1 / 2^{*}\right)\right)^{-1}$ so that $\gamma_{0} \in(0,2)$ and (3.7) are also satisfied.

Let $\gamma=1 /\left(2-\gamma_{0}\right)$ so that $\gamma \gamma_{0}=2 \gamma-1$. Then, by Hölder's inequality we have using (3.7)

$$
\begin{gathered}
\int b_{j} w^{2} \eta^{2 \gamma-1} \partial_{j} \eta \leq \int\left|b_{j}\right|\left|w \eta^{\gamma}\right|^{\gamma_{0}}|w|^{2-\gamma_{0}}\left|\partial_{j} \eta\right| \\
\leq\|b\|_{L^{\bar{q}}}\left\|w \eta^{\gamma}\right\|_{L^{2^{*}}}^{\gamma_{0}}\left\|w|\nabla \eta|^{1 /\left(2-\gamma_{0}\right)}\right\|_{L^{2}}^{2-\gamma_{0}}
\end{gathered}
$$

as $0 \leq \eta \leq 1$. By the Gagliardo-Nirenberg inequality, this leads to

$$
\int b_{j} w^{2} \eta^{2 \gamma-1} \partial_{j} \eta \leq \frac{1}{2}\left\|\nabla\left(w \eta^{\gamma}\right)\right\|_{L^{2}}^{2}+C\|b\|_{L^{\bar{q}}}^{2 /\left(2-\gamma_{0}\right)}\left\|w|\nabla \eta|^{1 /\left(2-\gamma_{0}\right)}\right\|_{L^{2}}^{2} .
$$

By (3.6), (3.4), and (3.9), we obtain

$$
\begin{aligned}
\int\left|\nabla\left(u^{\beta / 2+1} \eta^{\gamma}\right)\right|^{2} \leq C & \int u^{\beta+2} \eta^{2 \gamma-1}|\Delta \eta|+C \int u^{\beta+2} \eta^{2 \gamma-2}|\nabla \eta|^{2} \\
& +C\|b\|_{L^{\bar{q}}}^{2 /\left(2-\gamma_{0}\right)}\left\|u^{\beta / 2+1}(\nabla \eta)^{1 /\left(2-\gamma_{0}\right)}\right\|_{L^{2}}^{2} .
\end{aligned}
$$

By Sobolev embedding used in the left side of (3.10), we get

$$
\begin{aligned}
\left\|u^{\beta / 2+1} \eta^{\gamma}\right\|_{L^{2 \chi}} \leq C & \left(\int u^{\beta+2} \eta^{2 \gamma-1}|\Delta \eta|\right)^{1 / 2}+C\left(\int u^{\beta+2} \eta^{2 \gamma-2}|\nabla \eta|^{2}\right)^{1 / 2} \\
& +C\|b\|_{L^{\bar{q}}}^{1 /\left(2-\gamma_{0}\right)}\left\|u^{\beta / 2+1}(\nabla \eta)^{1 /\left(2-\gamma_{0}\right)}\right\|_{L^{2}},
\end{aligned}
$$

where $\chi=n /(n-2)$ if $n \geq 3$ and $\chi>2$ is arbitrary if $n=2$. Now, let $\eta \in C_{0}^{\infty}(\Omega)$ be such that $\eta \equiv 1$ in $B_{r_{i+1}}, \eta \equiv 0$ in $B_{r_{i}}^{c},|\nabla \eta| \leq C /\left(r_{i}-r_{i+1}\right)$, and $|\Delta \eta| \leq C /\left(r_{i}-r_{i+1}\right)^{2}$. Then we have

$$
\begin{aligned}
\left\|u^{\beta / 2+1}\right\|_{L^{2 \chi}\left(B_{r_{i+1}}\right)} \leq & \frac{C}{r_{i}-r_{i+1}}\left\|u^{\beta / 2+1}\right\|_{L^{2}\left(B_{r_{i}}\right)} \\
& \quad+\frac{C}{\left(r_{i}-r_{i+1}\right)^{1 /\left(2-\gamma_{0}\right)}}\|b\|_{L^{\bar{q}}\left(B_{r_{i}}\right)}^{1 /\left(2-\gamma_{0}\right)}\left\|u^{\beta / 2+1}\right\|_{L^{2}\left(B_{r_{i}}\right)} .
\end{aligned}
$$

The main point of (3.12) is that, since $\chi>1$, we have a bound on a higher norm of $u$ on a smaller ball in terms of the lower norm of $u$ on a larger ball. We now apply the estimate (3.12) iteratively on pairs of balls $B_{r_{i+1}} \subset B_{r_{i}}$, and also let $\beta_{i} \rightarrow+\infty$. More precisely, we choose $\beta_{i}=2\left(\chi^{i}-1\right)$ and $r_{i}=\theta+(\tau-\theta) 2^{-i}$ for $i=0,1,2, \ldots$, so that $r_{i}-r_{i+1}=(\tau-\theta) 2^{-(i+1)}$. We obtain

$$
\begin{aligned}
& \|u\|_{L^{2 \chi^{i+1}}\left(B_{r_{i+1}}\right)} \\
\leq & \left(\frac{C 2^{i+1}}{\tau-\theta}+\left(\frac{C 2^{i+1}}{\tau-\theta}\|b\|_{L^{\bar{q}}\left(B_{r_{i}}\right)}\right)^{1 /\left(2-\gamma_{0}\right)}\right)^{1 / \chi^{i}}\|u\|_{L^{2 \chi^{i}\left(B_{r_{i}}\right)}} \\
\leq & C^{1 / \chi^{i}} 2^{(i+1) /\left(\gamma_{1} \chi^{i}\right)}\left((\tau-\theta)^{-1}+\left((\tau-\theta)^{-1}\|b\|_{L^{\bar{q}}\left(B_{r_{i}}\right)}\right)^{1 /\left(2-\gamma_{0}\right)}\right)^{1 / \chi^{i}}\|u\|_{L^{2 \chi^{i}}\left(B_{r_{i}}\right)},
\end{aligned}
$$


where $\gamma_{1}=\min \left\{2-\gamma_{0}, 1\right\}$. By iteration, letting $i \rightarrow+\infty$, we conclude that the estimate (2.4) holds for $p \geq 2$.

Now, let $p \in(0,2)$. We have just shown that

$$
\begin{aligned}
\sup _{B_{\theta}} u & \leq C\left((\tau-\theta)^{-1}+\left((\tau-\theta)^{-1}\|b\|_{L^{\bar{q}}\left(B_{\tau}\right)}\right)^{1 /\left(2-\gamma_{0}\right)}\right)^{n / 2}\|u\|_{L^{2}\left(B_{\tau}\right)} \\
& \leq C\left((\tau-\theta)^{-1}+\left((\tau-\theta)^{-1}\|b\|_{L^{\bar{q}}\left(B_{\tau}\right)}\right)^{1 /\left(2-\gamma_{0}\right)}\right)^{n / 2}\|u\|_{L^{\infty}\left(B_{\tau}\right)}^{1-p / 2}\|u\|_{L^{p}\left(B_{\tau}\right)}^{p / 2},
\end{aligned}
$$

which leads to

$$
\sup _{B_{\theta}} u \leq \frac{1}{2}\|u\|_{L^{\infty}\left(B_{\tau}\right)}+C\left((\tau-\theta)^{-1}+\left((\tau-\theta)^{-1}\|b\|_{L^{\bar{q}}\left(B_{\tau}\right)}\right)^{1 /\left(2-\gamma_{0}\right)}\right)^{n / p}\|u\|_{L^{p}\left(B_{\tau}\right)} .
$$

A standard iteration argument (cf. [8, Lemma 4.3]) then implies

$$
\sup _{B_{\theta}} u \leq C\left((\tau-\theta)^{-1}+\left((\tau-\theta)^{-1}\|b\|_{L^{\bar{q}}\left(B_{\tau}\right)}\right)^{1 /\left(2-\gamma_{0}\right)}\right)^{n / p}\|u\|_{L^{p}\left(B_{\tau}\right)},
$$

and the proof of Lemma 2.4 is complete.

\section{Proof of Lemma 2.5}

We assume without loss of generality that $R=1$. The proof is similar in spirit to that of Lemma 2.4: We obtain an a priori bound and use it iteratively.

Let $u$ be a nonnegative Lipschitz supersolution to (1.1). We assume without loss of generality that $u>0$, and consider $v=1 / u$. The function $v$ satisfies

$$
-\Delta v+b \cdot \nabla v-a v \leq 0 \quad \text { in } \Omega
$$

or equivalently

$$
\int\left(\partial_{j} v\right)\left(\partial_{j} \varphi\right)+\int b_{j}\left(\partial_{j} v\right) \varphi-\int a v \varphi \leq 0
$$

for any function $\varphi \in C_{0}^{\infty}(\Omega)$ such that $\varphi \geq 0$ in $\Omega$. By Lemma 2.4, it follows that for any $0<\theta<\tau<1$ and $p>0$, we have

$$
\sup _{B_{\theta}} v \leq C\|v\|_{L^{p}\left(B_{\tau}\right)}
$$

with $C=C\left(n, p, q, \bar{q}, \tau, \theta, M_{1}\right)$. Therefore, we have

$$
\inf _{B_{\theta}} u \geq \frac{1}{C}\left(\int_{B_{\tau}} u^{-p} \int_{B_{\tau}} u^{p}\right)^{-1 / p}\left(\int_{B_{\tau}} u^{p}\right)^{1 / p} .
$$

We claim that there exists $p_{0}>0$ such that

$$
\int_{B_{\tau}} u^{-p_{0}} \int_{B_{\tau}} u^{p_{0}} \leq C
$$

with a constant $C=C\left(n, q, \bar{q}, \tau, M_{1}\right)$, which would finish the proof of Lemma 2.5. 
Reduction to an exponential bound. In order to prove (4.5) for some sufficiently small $p_{0}>0$, denote

$$
(\log u)_{B_{\tau}}=\frac{1}{\left|B_{\tau}\right|} \int_{B_{\tau}} \log u
$$

and set

$$
w=\log u-(\log u)_{B_{\tau}} .
$$

We shall show that there exists $p_{0}>0$ such that

$$
\int_{B_{\tau}} e^{p_{0}|w|} \leq C
$$

where $C=C(\tau)$, which in turn implies (4.5). Indeed, if we assume that (4.7) holds, then

$$
\int_{B_{\tau}} e^{p_{0}\left(\log u-(\log u)_{B_{\tau}}\right)} \leq C
$$

and

$$
\int_{B_{\tau}} e^{-p_{0}\left(\log u-(\log u)_{B_{\tau}}\right)} \leq C .
$$

Therefore, we have $e^{-p_{0}(\log u)_{B_{\tau}}} \int_{B_{\tau}} e^{p_{0} \log u} \leq C$ and $e^{p_{0}(\log u)_{B_{\tau}}} \int_{B_{\tau}} e^{-p_{0} \log u} \leq C$. Multiplying these two inequalities then leads to (4.5).

An $L^{2}$-bound for $w$. We now prove (4.7). First, we establish bounds on the $L^{2}$-norm of $w$. The function $w$ satisfies

$$
|\nabla w|^{2} \leq-\Delta w+b \cdot \nabla w+a \quad \text { in } B_{1} .
$$

Fix $\tau \in(0,1)$, and let $(1+\tau) / 2 \leq r_{1}<r_{2} \leq 1$. Also, let $\eta \in C_{0}^{1}(\Omega)$ with $0 \leq \eta \leq 1$ be a cutoff such that $\eta \equiv 1$ on $B_{r_{1}}, \eta \equiv 0$ on $B_{r_{2}}^{c}$, and $|\nabla \eta| \leq C /\left(r_{2}-r_{1}\right)$. Multiplying (4.10) by $\eta^{2}$ and integrating over $B_{1}$, we obtain

$$
\begin{aligned}
\int_{B_{1}}|\nabla w|^{2} \eta^{2} & \leq 2 \int_{B_{1}}\left(\partial_{j} w\right) \eta\left(\partial_{j} \eta\right)+\int_{B_{1}} b_{j}\left(\partial_{j} w\right) \eta^{2}+\int_{B_{1}} a \eta^{2} \\
& \leq 2\|\eta \nabla w\|_{L^{2}}\|\nabla \eta\|_{L^{2}}+\int_{B_{1}} b_{j}\left(\partial_{j} w\right) \eta^{2}+\|a\|_{L^{q}}\left\|\eta^{2}\right\|_{L^{q^{\prime}}}
\end{aligned}
$$

where $1 / q+1 / q^{\prime}=1$. Integrating by parts in the drift term and using $\operatorname{div} b=0$ gives

$$
\int_{B_{1}} b_{j} \partial_{j} w \eta^{2}=-2 \int_{B_{1}} b_{j} w \eta \partial_{j} \eta \leq 2\|b\|_{L^{\bar{q}}}\|w \eta\|_{L^{\bar{q}^{\prime}}}\|\nabla \eta\|_{L^{\infty}},
$$

where $1 / \bar{q}+1 / \bar{q}^{\prime}=1$. By the Gagliardo-Nirenberg inequality, we have

$$
\|w \eta\|_{L^{\bar{q}^{\prime}}} \leq C\|w \eta\|_{L^{2}}^{1-\alpha}\|\nabla(w \eta)\|_{L^{2}}^{\alpha}
$$

with $\alpha=n / 2-n / \bar{q}^{\prime}$ if $\bar{q}^{\prime} \geq 2$ and $\alpha=0$ otherwise. Using Young's inequality and

$$
\|\nabla(w \eta)\|_{L^{2}}^{\alpha} \leq C\|\eta \nabla w\|_{L^{2}}^{\alpha}+C\|w \nabla \eta\|_{L^{2}}^{\alpha},
$$


we obtain

$$
\begin{gathered}
\int_{B_{1}} b_{j} \partial_{j} w \eta^{2} \leq \frac{1}{4}\|\eta \nabla w\|_{L^{2}}^{2}+C\|b\|_{L^{\bar{q}}}^{2 /(2-\alpha)}\|w \eta\|_{L^{2}}^{(2-2 \alpha) /(2-\alpha)}\|\nabla \eta\|_{L^{\infty}}^{2 /(2-\alpha)} \\
+C\|b\|_{L^{\bar{q}}}\|w \eta\|_{L^{2}}^{1-\alpha}\|w \nabla \eta\|_{L^{2}}^{\alpha}\|\nabla \eta\|_{L^{\infty}}
\end{gathered}
$$

From (4.11) and (4.15), it follows that

$$
\begin{aligned}
\|\eta \nabla w\|_{L^{2}}^{2} \leq & \frac{1}{2}\|\eta \nabla w\|_{L^{2}}^{2}+\frac{C}{\left(r_{2}-r_{1}\right)^{2}} \\
& +\frac{C}{\left(r_{2}-r_{1}\right)^{2 /(2-\alpha)}}\|b\|_{L^{\bar{q}}}^{2 /(2-\alpha)}\|w \eta\|_{L^{2}}^{(2-2 \alpha) /(2-\alpha)} \\
& +\frac{C}{\left(r_{2}-r_{1}\right)^{1+\alpha}}\|b\|_{L^{\bar{q}}}\|w\|_{L^{2}}+C\|a\|_{L^{q}}
\end{aligned}
$$

Absorbing the factor $\|\eta \nabla w\|_{L^{2}}^{2}$ and using Young's inequality, we get

$$
\begin{aligned}
\|\nabla w\|_{L^{2}\left(B_{r_{1}}\right)}^{2} & \leq \frac{1}{2}\|w\|_{L^{2}\left(B_{r_{2}}\right)}^{2}+\frac{C}{\left(r_{2}-r_{1}\right)^{2+2 \alpha}}\|b\|_{L^{\bar{q}}}^{2}+C\|a\|_{L^{q}}+\frac{C}{\left(r_{2}-r_{1}\right)^{2}} \\
& \leq \frac{1}{2}\|w\|_{L^{2}\left(B_{r_{2}}\right)}^{2}+\frac{C M_{1}}{\left(r_{2}-r_{1}\right)^{2+2 \alpha}},
\end{aligned}
$$

where $M_{1}=1+\|a\|_{L^{q}\left(B_{1}\right)}+\|b\|_{L^{\bar{q}}\left(B_{1}\right)}^{2}$. Now, since

$$
\int_{B_{\tau}} w=0
$$

by the Poincaré inequality and (4.17), we obtain

$$
\|\nabla w\|_{L^{2}\left(B_{r_{1}}\right)}^{2} \leq \frac{1}{2}\|\nabla w\|_{L^{2}\left(B_{r_{2}}\right)}^{2}+\frac{C M_{1}}{\left(r_{2}-r_{1}\right)^{2+2 \alpha}}
$$

for all $(1+\tau) / 2 \leq r_{1}<r_{2} \leq 1$, which implies (cf. [8, Lemma 4.3])

$$
\int_{B_{(1+\tau) / 2}}|\nabla w|^{2} \leq C_{\tau} M_{1}
$$

where the constant $C_{\tau}$ depends on $\tau \in(0,1)$. Also, since $(1+\tau) / 2 \geq \tau$, we conclude by the Poincaré inequality

$$
\int_{B_{(1+\tau) / 2}} w^{2} \leq C \int_{B_{(1+\tau) / 2}}|\nabla w|^{2} \leq C_{\tau} M_{1} .
$$

Bounds on the higher norms of $w$. Next, we need to estimate $\int_{B_{\tau}}|w|^{\beta}$ for all $\beta \geq 1$. As in the proof of Lemma 2.4 the idea is to bound first the higher norms of $w$ on smaller balls in terms of the lower norms of $w$ on larger balls and then use the iteration process.

We multiply (4.10) by $|w|^{2 \beta} \eta^{2 \gamma}$ and integrate over $B_{1}$ in order to obtain

$$
\int_{B_{1}}|w|^{2 \beta}|\nabla w|^{2} \eta^{2 \gamma} \leq 2 \beta \int_{B_{1}}|w|^{2 \beta-2} w|\nabla w|^{2} \eta^{2 \gamma}+2 \gamma \int_{B_{1}}|w|^{2 \beta}\left(\partial_{j} w\right) \eta^{2 \gamma-1}\left(\partial_{j} \eta\right)
$$




$$
-\frac{2 \gamma}{2 \beta+1} \int_{B_{1}} b_{j}|w|^{2 \beta} w \eta^{2 \gamma-1}\left(\partial_{j} \eta\right)+\int_{B_{1}} a|w|^{2 \beta} \eta^{2 \gamma} .
$$

Here we utilized $\operatorname{div} b=0$ and $\partial_{j}|w|=w \partial_{j} w /|w|$. For the first term on the right side of (4.21) we use

$$
2 \beta|w|^{2 \beta-1} \leq \frac{1}{4}|w|^{2 \beta}+(8 \beta)^{2 \beta},
$$

while for the second

$$
\begin{aligned}
& 2 \gamma \int_{B_{1}}|w|^{2 \beta}\left(\partial_{j} w\right) \eta^{2 \gamma-1}\left(\partial_{j} \eta\right) \\
& \quad \leq \frac{1}{4} \int_{B_{1}}|w|^{2 \beta}|\nabla w|^{2} \eta^{2 \gamma}+C \gamma^{2} \int_{B_{1}}|w|^{2 \beta} \eta^{2 \gamma-2}|\nabla \eta|^{2} .
\end{aligned}
$$

This leads to

$$
\begin{aligned}
\int_{B_{1}}|w|^{2 \beta}|\nabla w|^{2} \eta^{2 \gamma} \leq C & (8 \beta)^{2 \beta} \int_{B_{1}}|\nabla w|^{2} \eta^{2 \gamma}+C \gamma^{2} \int_{B_{1}}|w|^{2 \beta} \eta^{2 \gamma-2}|\nabla \eta|^{2} \\
& +\frac{C \gamma}{\beta+1} \int_{B_{1}}|b||w|^{2 \beta+1} \eta^{2 \gamma-1}|\nabla \eta|+C \int_{B_{1}}|a||w|^{2 \beta} \eta^{2 \gamma} .
\end{aligned}
$$

Let $\tau \leq r<R \leq(1+\tau) / 2$. We now choose a cutoff $\eta \in C_{0}^{1}(\Omega)$ with $0 \leq \eta \leq 1$ such that $\eta \equiv 1$ on $B_{r}, \eta \equiv 0$ on $B_{R}^{c}$, and $|\nabla \eta| \leq C /(R-r)$. By (4.17), we have for the first term on the right side of $(4.24)$

$$
(8 \beta)^{2 \beta} \int_{B_{1}}|\nabla w|^{2} \eta^{2 \gamma} \leq(8 \beta)^{2 \beta} \int_{B_{(1+\tau) / 2}}|\nabla w|^{2} \leq C_{\tau}(8 \beta)^{2 \beta} M_{1} .
$$

On the other hand, for the left side of (4.24), we use

$$
\left|\nabla\left(|w|^{\beta+1} \eta^{\gamma}\right)\right|^{2} \leq 2 \gamma^{2}|w|^{2 \beta+2} \eta^{2 \gamma-2}|\nabla \eta|^{2}+2(\beta+1)^{2}|w|^{2 \beta}|\nabla w|^{2} \eta^{2 \gamma} .
$$

Hence, we obtain

$$
\begin{aligned}
\int_{B_{1}}\left|\nabla\left(|w|^{\beta+1} \eta^{\gamma}\right)\right|^{2} \leq & C \gamma^{2} \int_{B_{1}}|w|^{2 \beta+2} \eta^{2 \gamma-2}|\nabla \eta|^{2}+C(\beta+1)^{2}(8 \beta)^{2 \beta} M_{1} \\
& +C \gamma^{2}(\beta+1)^{2} \int_{B_{1}}|w|^{2 \beta} \eta^{2 \gamma-2}|\nabla \eta|^{2} \\
& +C \gamma(\beta+1) \int_{B_{1}}|b||w|^{2 \beta+1} \eta^{2 \gamma-1}|\nabla \eta| \\
& +C(\beta+1)^{2} \int_{B_{1}}|a||w|^{2 \beta} \eta^{2 \gamma}
\end{aligned}
$$

For the third term on the right side we utilize

$$
(\beta+1)^{2}|w|^{2 \beta} \leq \frac{(\beta+1)^{2 \beta+2}}{\beta+1}+\frac{\left(|w|^{2 \beta}\right)^{(\beta+1) / \beta}}{(\beta+1) / \beta} \leq(8 \beta)^{2 \beta}+|w|^{2 \beta+2},
$$

which gives

$$
C \gamma^{2}(\beta+1)^{2} \int_{B_{1}}|w|^{2 \beta} \eta^{2 \gamma-2}|\nabla \eta|^{2}
$$




$$
\begin{aligned}
& \leq C(8 \beta)^{2 \beta} \gamma^{2} \int_{B_{1}} \eta^{2 \gamma-2}|\nabla \eta|^{2}+C \gamma^{2} \int_{B_{1}}|w|^{2 \beta+2} \eta^{2 \gamma-2}|\nabla \eta|^{2} \\
& \leq \frac{C(8 \beta)^{2 \beta} \gamma^{2} M_{1}}{(R-r)^{2}}+C \gamma^{2} \int_{B_{1}}|w|^{2 \beta+2} \eta^{2 \gamma-2}|\nabla \eta|^{2}
\end{aligned}
$$

as $M_{1} \geq 1$. The last two terms in (4.27) are estimated as follows. First, we have

$$
\begin{aligned}
\int_{B_{1}}|a||w|^{2 \beta} \eta^{2 \gamma} & =\int_{B_{1}}|a|\left(|w|^{\beta+1} \eta^{\gamma}\right)^{2 \beta /(\beta+1)} \eta^{2 \gamma /(\beta+1)} \\
& \leq\|a\|_{L^{q}}\left\||w|^{\beta+1} \eta^{\gamma}\right\|_{L^{2 \beta q^{\prime} /(\beta+1)}}^{2 \beta /(\beta+1)},
\end{aligned}
$$

where $1 / q+1 / q^{\prime}=1$. Now, we use the Gagliardo-Nirenberg inequality

$$
\left\||w|^{\beta+1} \eta^{\gamma}\right\|_{L^{2 \beta q^{\prime} /(\beta+1)}} \leq C\left\||w|^{\beta+1} \eta^{\gamma}\right\|_{L^{2}}^{1-\alpha}\left\|\nabla\left(|w|^{\beta+1} \eta^{\gamma}\right)\right\|_{L^{2}}^{\alpha}
$$

with $\alpha=n / 2-n /\left(2 \beta q^{\prime} /(\beta+1)\right)$ if $2 \beta q^{\prime} /(\beta+1) \geq 2$, and $\alpha=0$ otherwise. Note that by the assumption $q>n / 2$ we have $\alpha<1$. If $\alpha>0$ we obtain by Young's inequality

$$
\begin{aligned}
& \quad \int_{B_{1}}|a||w|^{2 \beta} \eta^{2 \gamma} \leq C\|a\|_{L^{q}}\left\||w|^{\beta+1} \eta^{\gamma}\right\|_{L^{2}}^{2(1-\alpha) \beta /(\beta+1)}\left\|\nabla\left(|w|^{\beta+1} \eta^{\gamma}\right)\right\|_{L^{2}}^{2 \alpha \beta /(\beta+1)} \\
& \leq\left(\frac{1}{(2(\beta+1))^{2 \alpha \beta /(\beta+1)}}\left\|\nabla\left(|w|^{\beta+1} \eta^{\gamma}\right)\right\|_{L^{2}}^{2 \alpha \beta /(\beta+1)}\right)^{(\beta+1) / \alpha \beta} \\
& \quad+C\left((2(\beta+1))^{2 \alpha \beta /(\beta+1)}\|a\|_{L^{q}}\left\||w|^{\beta+1} \eta^{\gamma}\right\|_{L^{2}}^{2(1-\alpha) \beta /(\beta+1)}\right)^{(\beta+1) /(\beta(1-\alpha)+1)}
\end{aligned}
$$

If $\alpha=0$ the same inequality holds with the first term on the far right side omitted. As $\alpha \in[0,1)$ and $2 \alpha \beta /(\beta+1) \leq 2$, this implies

$$
\begin{aligned}
\int_{B_{1}}|a||w|^{2 \beta} \eta^{2 \gamma} \leq & \frac{1}{(2(\beta+1))^{2}}\left\|\nabla\left(|w|^{\beta+1} \eta^{\gamma}\right)\right\|_{L^{2}}^{2} \\
& +C(\beta+1)^{2 \alpha_{1}}\|a\|_{L^{q}}^{\alpha_{1}}\left\||w|^{\beta+1} \eta^{\gamma}\right\|_{L^{2}}^{\alpha_{2}}
\end{aligned}
$$

Here we denoted $\alpha_{1}=(\beta+1) /(\beta(1-\alpha)+1)$ and $\alpha_{2}=2 \beta(1-\alpha) /(\beta(1-\alpha)+1)$. Observe that $\alpha_{1} \geq 1$ and $\alpha_{1}$ is smaller than a constant independent of $\beta$, while $0<\alpha_{2}<2$ with $\alpha_{2} \rightarrow 2$ as $\beta \rightarrow \infty$.

For the last remaining term in (4.27), we have

$$
\begin{aligned}
& C \gamma(\beta+1) \int_{B_{1}}|b||w|^{2 \beta+1} \eta^{2 \gamma-1}|\nabla \eta| \\
= & C \gamma(\beta+1) \int_{B_{1}}|b|\left(|w|^{\beta+1} \eta^{\gamma}\right)^{(2 \beta+1) /(\beta+1)} \eta^{\gamma /(\beta+1)-1}|\nabla \eta| .
\end{aligned}
$$

Let us choose $\gamma=\beta+1$. Then, the above expression becomes

$$
\begin{aligned}
& C \gamma(\beta+1) \int_{B_{1}}|b|\left(|w|^{\beta+1} \eta^{\gamma}\right)^{(2 \beta+1) /(\beta+1)}|\nabla \eta| \\
\leq & C(\beta+1)^{2}\|b\|_{L^{\bar{q}}}\left\||w|^{\beta+1} \eta^{\gamma}\right\|_{L^{(2 \beta+1) \bar{q}^{\prime} /(\beta+1)}}^{(2 \beta+1) /(\beta+1)}\|\nabla \eta\|_{L^{\infty}},
\end{aligned}
$$


where $1 / \bar{q}+1 / \bar{q}^{\prime}=1$. Once again we apply the Gagliardo-Nirenberg inequality

$$
\left\||w|^{\beta+1} \eta^{\gamma}\right\|_{L^{(2 \beta+1) \bar{q}^{\prime} /(\beta+1)}} \leq C\left\||w|^{\beta+1} \eta^{\gamma}\right\|_{L^{2}}^{1-\bar{\alpha}}\left\|\nabla\left(|w|^{\beta+1} \eta^{\gamma}\right)\right\|_{L^{2}}^{\bar{\alpha}}
$$

with $\bar{\alpha}=n / 2-n /\left((2 \beta+1) \bar{q}^{\prime} /(\beta+1)\right)$ if $(2 \beta+1) \bar{q}^{\prime} /(\beta+1) \geq 2$ and $\bar{\alpha}=0$ otherwise.

Thus, by Young's inequality, we have

$$
\begin{gathered}
\quad C \gamma(\beta+1) \int_{B_{1}}|b|\left(|w|^{\beta+1} \eta^{\gamma}\right)^{(2 \beta+1) /(\beta+1)}|\nabla \eta| \\
\leq C(\beta+1)^{2}\|b\|_{L^{\bar{q}}}\left\||w|^{\beta+1} \eta^{\gamma}\right\|_{L^{2}}^{(1-\bar{\alpha})(2 \beta+1) /(\beta+1)} \\
\quad \times\left\|\nabla\left(|w|^{\beta+1} \eta^{\gamma}\right)\right\|_{L^{2}}^{\bar{\alpha}(2 \beta+1) /(\beta+1)}\|\nabla \eta\|_{L^{\infty}} \\
\leq \frac{1}{4}\left\|\nabla\left(|w|^{\beta+1} \eta^{\gamma}\right)\right\|_{L^{2}}^{2}+\frac{C(\beta+1)^{2 \bar{\alpha}_{1}}}{(R-r)^{\bar{\alpha}_{1}}}\|b\|_{L^{\frac{q}{q}}}^{\bar{\alpha}_{1}}\left\|\left.w\right|^{\beta+1} \eta^{\gamma}\right\|_{L^{2}}^{\bar{\alpha}_{2} .}
\end{gathered}
$$

Here we denoted $\bar{\alpha}_{1}=(2 \beta+2) /(2 \beta(1-\bar{\alpha})+2-\bar{\alpha})$ and $\bar{\alpha}_{2}=2(2 \beta+1)(1-\bar{\alpha}) /(2 \beta(1-$ $\bar{\alpha})+2-\bar{\alpha})$. Note that, as in (4.31), we have $\bar{\alpha}_{1} \geq 1$ and $\bar{\alpha}_{1}$ is less than a constant independent of $\beta$, while $0<\bar{\alpha}_{2}<2$, and $\bar{\alpha}_{2} \rightarrow 2$ when $\beta \rightarrow \infty$.

Putting together (4.27), (4.29), (4.31), and (4.37), we obtain

$$
\begin{gathered}
\left\|\nabla\left(|w|^{\beta+1} \eta^{\gamma}\right)\right\|_{L^{2}\left(B_{R}\right)}^{2} \leq \frac{C(\beta+1)^{2}}{(R-r)^{2}}\left\||w|^{\beta+1}\right\|_{L^{2}\left(B_{R}\right)}^{2}+\frac{C(\beta+1)^{2}(8 \beta)^{2 \beta} M_{1}}{(R-r)^{2}} \\
+C(\beta+1)^{2 \alpha_{1}+2}\|a\|_{L^{q}\left(B_{R}\right)}^{\alpha_{1}}\left\||w|^{\beta+1}\right\|_{L^{2}\left(B_{R}\right)}^{\alpha_{2}} \\
+\frac{C(\beta+1)^{2 \bar{\alpha}_{1}}}{(R-r)^{\bar{\alpha}_{1}}}\|b\|_{L^{\bar{q}\left(B_{R}\right)}}^{\bar{\alpha}_{1}}\left\||w|^{\beta+1}\right\|_{L^{2}\left(B_{R}\right)}^{\bar{\alpha}_{2}} .
\end{gathered}
$$

Using Sobolev embedding, we may rewrite (4.38) in the form

$$
\begin{aligned}
\left\||w|^{\beta+1}\right\|_{L^{2 \chi}\left(B_{r}\right)}^{2} \leq \frac{C(\beta+1)^{2 \kappa} M_{1}^{\tilde{\alpha}}}{(R-r)^{\bar{\alpha}_{1}+2}}\left(\left\||w|^{\beta+1}\right\|_{L^{2}\left(B_{R}\right)}^{2}+(8 \beta)^{2 \beta}\right. & \\
& \left.+\left\||w|^{\beta+1}\right\|_{L^{2}\left(B_{R}\right)}^{\alpha_{2}}+\left\||w|^{\beta+1}\right\|_{L^{2}\left(B_{R}\right)}^{\bar{\alpha}_{2}}\right)
\end{aligned}
$$

where $\kappa=\max \left\{\alpha_{1}+1, \bar{\alpha}_{1}\right\}, \tilde{\alpha}=\max \left\{\alpha_{1}, \bar{\alpha}_{1} / 2\right\}$, and $\chi=n /(n-2)$ if $n \geq 3$ and $\chi>1$ if $n=2$. The estimate (4.39) is analogous to (3.12): A higher norm of $w$ on a smaller ball is bounded in terms of a lower norm of $w$ on a larger ball.

The case $0 \leq \beta<1$. We now show that (4.39) remains valid for $0 \leq \beta<1$ by considering two separate cases: $0 \leq \beta<1 / 2$ and $1 / 2 \leq \beta<1$. The estimates obtained here are similar to the ones already derived for $\beta \geq 1$, and we point out only the main steps.

First, for $0 \leq \beta<1 / 2$, we use as a test function $\left(|w|^{2}+1\right)^{\beta-1} w^{2} \eta^{2 \gamma}$ for (4.10) in order to obtain

$$
\begin{aligned}
\int_{B_{1}} \frac{w^{2}|\nabla w|^{2}}{\left(|w|^{2}+1\right)^{1-\beta}} \eta^{2 \gamma} \leq C & \int_{B_{1}} \frac{w|\nabla w|^{2}}{\left(|w|^{2}+1\right)^{1-\beta}} \eta^{2 \gamma}+2 \gamma \int_{B_{1}} \frac{w^{2} \partial_{j} w}{\left(|w|^{2}+1\right)^{1-\beta}} \eta^{2 \gamma-1} \partial_{j} \eta \\
& +C \gamma \int_{B_{1}}|b|\left(|w|^{2}+1\right)^{\beta+1 / 2} \eta^{2 \gamma-1} \partial_{j} \eta \\
& +\int_{B_{1}}|a|\left(|w|^{2}+1\right)^{\beta-1} w^{2} \eta^{2 \gamma}
\end{aligned}
$$


where for the drift term we also utilized $\operatorname{div} b=0$ and

$$
\int b_{j} \partial_{j} w\left(|w|^{2}+1\right)^{\beta-1} w^{2} \eta^{2 \gamma}=\int b_{j} \partial_{j} \phi \eta^{2 \gamma}=-2 \gamma \int b_{j} \phi \eta^{2 \gamma-1} \partial_{j} \eta
$$

with $\phi(x)=\int_{0}^{x}\left(y^{2}+1\right)^{\beta-1} y^{2} d y \leq C\left(x^{2}+1\right)^{\beta+1 / 2}$. Now, since $w \leq\left(|w|^{2}+1\right)^{1-\beta}$ for $0<$ $\beta<1 / 2$, we have by (4.19)

$$
\int_{B_{1}} \frac{w|\nabla w|^{2}}{\left(|w|^{2}+1\right)^{1-\beta}} \eta^{2 \gamma} \leq \int_{B_{1}}|\nabla w|^{2} \eta^{2 \gamma} \leq C M_{1}
$$

For the second term on the right side of (4.40), we get

$$
\begin{aligned}
& 2 \gamma \int_{B_{1}} \frac{w^{2} \partial_{j} w}{\left(|w|^{2}+1\right)^{1-\beta}} \eta^{2 \gamma-1} \partial_{j} \eta \\
\leq & \frac{1}{2} \int_{B_{1}} \frac{w^{2}|\nabla w|^{2}}{\left(|w|^{2}+1\right)^{1-\beta}} \eta^{2 \gamma}+C \gamma^{2} \int_{B_{1}} \frac{w^{2} \eta^{2 \gamma-2}|\nabla \eta|^{2}}{\left(|w|^{2}+1\right)^{1-\beta}} \\
\leq & \frac{1}{2} \int_{B_{1}} \frac{w^{2}|\nabla w|^{2}}{\left(|w|^{2}+1\right)^{1-\beta}} \eta^{2 \gamma}+\frac{C M_{1}}{(R-r)^{2}},
\end{aligned}
$$

where we used $\left(|w|^{2}+1\right)^{1-\beta} \geq 1$ and (4.20). Since

$$
\begin{aligned}
& \left|\nabla\left(\left(|w|^{2}+1\right)^{(\beta+1) / 2} \eta^{\gamma}\right)\right|^{2} \\
\leq & 2 \gamma^{2}\left(|w|^{2}+1\right)^{\beta+1} \eta^{2 \gamma-2}|\nabla \eta|^{2}+2(\beta+1)^{2}\left(|w|^{2}+1\right)^{\beta-1} w^{2}|\nabla w|^{2} \eta^{2 \gamma},
\end{aligned}
$$

this leads to

$$
\begin{aligned}
\int_{B_{1}}\left|\nabla\left(\left(|w|^{2}+1\right)^{(\beta+1) / 2} \eta^{\gamma}\right)\right|^{2} \leq C & \int_{B_{1}}\left(|w|^{2}+1\right)^{\beta+1} \eta^{2 \gamma-2}|\nabla \eta|^{2}+\frac{C M_{1}}{(R-r)^{2}} \\
& +C \int_{B_{1}}|b|\left(|w|^{2}+1\right)^{\beta+1 / 2} \eta^{2 \gamma-1} \partial_{j} \eta \\
& +C \int_{B_{1}}|a|\left(|w|^{2}+1\right)^{\beta-1} w^{2} \eta^{2 \gamma}
\end{aligned}
$$

Using estimates similar to (4.33) and (4.37) for the last two terms, and Sobolev's inequality for the left side of (4.44), we obtain

$$
\begin{aligned}
& \quad\left\|\left(|w|^{2}+1\right)^{(\beta+1) / 2}\right\|_{L^{2 \chi}\left(B_{r}\right)}^{2} \\
& \leq \frac{C M_{1}^{\tilde{\alpha}}}{(R-r)^{\bar{\alpha}_{1}+2}}\left(\left\|\left(|w|^{2}+1\right)^{(\beta+1) / 2}\right\|_{L^{2}\left(B_{R}\right)}^{2}+1\right. \\
& \left.\quad+\left\|\left(|w|^{2}+1\right)^{(\beta+1) / 2}\right\|_{L^{2}\left(B_{R}\right)}^{\alpha_{2}}+\left\|\left(|w|^{2}+1\right)^{(\beta+1) / 2}\right\|_{L^{2}\left(B_{R}\right)}^{\bar{\alpha}_{2}}\right),
\end{aligned}
$$

where $\tilde{\alpha}, \alpha_{2}$, and $\bar{\alpha}_{2}$ are defined as above. Hence, we conclude (4.39) for $0<\beta<1 / 2$.

Finally, if $1 / 2 \leq \beta<1$, we use as a test function $\left(|w|^{2}+\epsilon\right)^{\beta-1} w^{2} \eta^{2 \gamma}$ for (4.10). Then, we proceed exactly as in the case of $\beta \geq 1$. We conclude (4.39) by letting $\epsilon \rightarrow 0$. 
The iteration process. Next, we consider the iteration process. Let $\beta_{i}=\chi^{i}-1$ and $r_{i}=\tau+(1-\tau) / 2^{i+1}$ for $i=0,1,2, \ldots$. From (4.39), we get

$$
\begin{gathered}
\left\||w|^{\chi^{i}}\right\|_{L^{2 \chi\left(B_{r_{i+1}}\right)}}^{2} \leq C \chi^{2 \kappa i} 2^{\left(\bar{\alpha}_{1}+2\right)(i+2)} M_{1}^{\tilde{\alpha}}\left(\left\||w|^{\chi^{i}}\right\|_{L^{2}\left(B_{r_{i}}\right)}^{2}+\left(8 \chi^{i}\right)^{2 \chi^{i}}\right. \\
\left.+\left\||w|^{\chi^{i}}\right\|_{L^{2}\left(B_{r_{i}}\right)}^{\alpha_{2}}+\left\||w|^{\chi^{i}}\right\|_{L^{2}\left(B_{r_{i}}\right)}^{\bar{\alpha}_{2}}\right) \\
\leq C \chi^{2 \kappa i} 2^{\left(\bar{\alpha}_{1}+2\right)(i+2)} M_{1}^{\tilde{\alpha}}\left(\left\||w|^{\chi^{i}}\right\|_{L^{2}\left(B_{r_{i}}\right)}^{2}+\left(8 \chi^{i}\right)^{2 \chi^{i}}\right)
\end{gathered}
$$

for all $i=0,1,2, \ldots$ For the second inequality in (4.46) we also used $\alpha_{2}, \bar{\alpha}_{2} \leq 2$, so

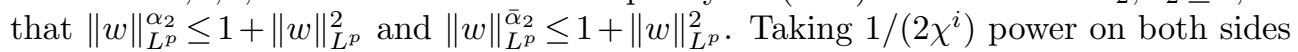
of (4.46) gives

$$
\begin{aligned}
& \|w\|_{L^{2 \chi^{i+1}}\left(B_{r_{i+1}}\right)} \\
\leq & C^{1 /\left(2 \chi^{i}\right)} \chi^{\kappa i / \chi^{i}} 2^{\left(\bar{\alpha}_{1}+2\right)(i+2) /\left(2 \chi^{i}\right)} M_{1}^{\tilde{\alpha} /\left(2 \chi^{i}\right)}\left(\|w\|_{L^{2 \chi^{i}\left(B_{r_{i}}\right)}}+8 \chi^{i}\right) .
\end{aligned}
$$

This leads to the inequality

$$
\|w\|_{L^{2 \chi^{i+1}\left(B_{r_{i+1}}\right)}} \leq\left(C M_{1}\right)^{\tilde{\alpha} /\left(2 \chi^{i}\right)}(2 \chi)^{\kappa(i+2) / \chi^{i}}\left(\|w\|_{L^{2 \chi^{i}\left(B_{r_{i}}\right)}}+8 \chi^{i}\right)
$$

for all $i=0,1,2, \ldots$, since $\tilde{\alpha} \geq 1$ and $\bar{\alpha}_{1}+2 \leq 2 \kappa$.

Note that if a sequence $Y_{i}$ satisfies $Y_{i+1} \leq C_{i}\left(Y_{i}+\chi^{i}\right)$ with $C_{i} \geq 1$ and $\prod_{i=1}^{\infty} C_{i} \leq \bar{K}$, then we have by induction

$$
Y_{i} \leq C_{1} C_{2} \ldots C_{i-1}\left(Y_{0}+\sum_{j=1}^{i} \chi^{j-1}\right) \leq \bar{K}\left(Y_{0}+\frac{\chi^{i}}{\chi-1}\right) \leq C\left(Y_{0}+\chi^{i}\right)
$$

for all $i=0,1,2, \ldots$. Thus, we obtain

$$
\|w\|_{L^{2 \chi^{i+1}\left(B_{r_{i+1}}\right)}} \leq C M_{1}^{C(n)}\left(C M_{1}+\chi^{i+1}\right) \leq C M_{1}^{C(n)} \chi^{i+1}
$$

for all $i=0,1,2, \ldots$, as $\sum_{j=1}^{i} j / \chi^{j} \leq C$.

Finally, for any $\beta \geq 1$ there exists $i \in\{0,1,2, \ldots\}$ such that

$$
2 \chi^{i} \leq \beta+1 \leq 2 \chi^{i+1} \text {. }
$$

Thus, we have

$$
\left(\int_{B_{\tau}}|w|^{\beta+1}\right)^{1 /(\beta+1)} \leq C\|w\|_{L^{2 \chi^{i+1}\left(B_{r_{i+1}}\right)}} \leq C M_{1}^{C(n)}(\beta+1) .
$$

Therefore, for all $\beta \geq 1$, we obtain

$$
\int_{B_{\tau}} \frac{\left(p_{0}|w|\right)^{(\beta+1)}}{(\beta+1) !} \leq p_{0}^{\beta+1}\left(C M_{1}^{C(n)} e\right)^{(\beta+1)} \leq \frac{1}{2^{(\beta+1)}}
$$

by taking

$$
p_{0}=\frac{1}{2 C M_{1}^{C(n)} e} .
$$


By (4.20), we also have

$$
\int_{B_{\tau}}|w| \leq C\left(\int_{B_{\tau}} w^{2}\right)^{1 / 2} \leq C M_{1}
$$

which gives (4.53) for $\beta \in[0,1]$ as well. It follows from (4.53) that (4.7) holds, and therefore the proof of the lemma is complete.

Acknowledgment. I.K. was supported in part by the NSF grants DMS-1009769 and DMS-1311943, L.R. was supported in part by the NSF grant DMS-0908507, and both M.I and L.R. were supported in part by NSF FRG grant DMS-115893.

\section{REFERENCES}

[1] H. Berestycki, A. Kiselev, A. Novikov, and L. Ryzhik, The explosion problem in a flow, J. Anal. Math., 110, 31-65, 2010.

[2] L.A. Caffarelli and A.F. Vasseur, The De Giorgi method for regularity of solutions of elliptic equations and its applications to fluid dynamics, Disc. Cont. Dyn. Sys. Ser. S, 3(3), 409$427,2010$.

[3] L.A. Caffarelli and A.F. Vasseur, Drift diffusion equations with fractional diffusion and the quasi-geostrophic equation, Ann. Math., 171, 1903-1930, 2010.

[4] P. Constantin, A. Kiselev, L. Ryzhik, and A. Zlatos, Diffusion and mixing in a fluid flow, Ann. Math., 68, 643-674, 2008.

[5] E. De Giorgi, Sulla differenziabilità e l'analiticità delle estremali degli integrali multipli regolari, Mem. Accad. Sci. Torino. Cl. Sci. Fis. Mat. Nat., 3(3), 25-43, 1957.

[6] S. Friedlander and V. Vicol, Global well-posedness for an advection-diffusion equation arising in magneto-geostrophic dynamics, Ann. Inst. H. Poincaré Anal. Non Linéaire, 28(2), 283301, 2011.

[7] M. Giaquinta, Introduction to Regularity Theory for Nonlinear Elliptic Systems, Lectures in Mathematics ETH Zürich, Birkhäuser Verlag, Basel, 1993.

[8] Q. Han and F. Lin, Elliptic Partial Differential Equations, Second Edition, Courant Lecture Notes in Mathematics, Courant Institute of Mathematical Sciences, New York, 1, 2011.

[9] I. Kukavica, On the dissipative scale for the Navier-Stokes equation, Indiana Univ. Math. J., 48(3), 1057-1081, 1999.

[10] G. Koch, N. Nadirashvili, G.A. Seregin, and V. Šverák, Liouville theorems for the Navier-Stokes equations and applications, Acta Math., 203(1), 83-105, 2009.

[11] J. Moser, A Harnack inequality for parabolic differential equations, Comm. Pure Appl. Math., 17, 101-134, 1964.

[12] J. Nash, Continuity of solutions of parabolic and elliptic equations, Amer. J. Math., 80, 931954, 1958.

[13] A.I. Nazarov and N.N. Ural'tseva, The Harnack inequality and related properties of solutions of elliptic and parabolic equations with divergence-free lower-order coefficients, Algebra i Analiz, 23(1), 136-168, 2011.

[14] G. Stampacchia, Le problème de Dirichlet pour les équations elliptiques du second ordre à coefficients discontinus, Ann. Inst. Fourier (Grenoble), 15(1), 189-258, 1965.

[15] G. Seregin, L. Silvestre, V. Šverák, and A. Zlatoš, On divergence-free drifts, J. Diff. Equ., 252(1), 505-540, 2012.

[16] Q.S. Zhang, A strong regularity result for parabolic equations, Comm. Math. Phys., 244(2), 245-260, 2004. 